\title{
Utilizing a High Definition Live Video Platform to Facilitate Public Service Delivery
}

\author{
Vishanth Weerakkody ${ }^{1}$, Ramzi El-Haddadeh ${ }^{1}$, \\ Ioannis P. Chochliouros ${ }^{2}$, and Donal Morris ${ }^{3}$ \\ ${ }^{1}$ Brunel University, \\ Kingston Lane, Uxbridge, UB8 3PH, Middlesex, United Kingdom \\ \{Vishanth.Weerakkody, Ramzi.El-Haddadeh\}@brunel.ac.uk \\ ${ }^{2}$ Research Programs Section, \\ Hellenic Telecommunications Organization (OTE) S.A., \\ 99 Kifissias Avenue, GR-151 24, Athens, Greece \\ ichochliouros@oteresearch.gr \\ ${ }^{3}$ RedZinc Services Ltd., \\ Taylor's Lane, Dublin 8, Dublin, Ireland \\ dmorrisaredzinc.net
}

\begin{abstract}
The LiveCity Project effort intends to create a city-based "Living Lab" and associated ecosystem to pilot live interactive high-definition video-tovideo $(\mathrm{v} 2 \mathrm{v})$ on ultrafast wireless and wireline Internet infrastructure for the support of appropriate public service use cases among a number of city user communities initially in four major European cities. The essential aim is to empower the citizens of a city to interact with each other in a more productive, efficient and socially useful way by using v $2 \mathrm{v}$ over the Internet, as the latter can be considered to improve city administration, enhance education and city experiences for tourists/cultural consumers and save patients' lives. We discuss the role that stakeholders can play in identifying appropriate KPIs to assess the progress of the LiveCity concept, covering the underlying network infrastructure, the intended services-facilities per specific case, as well as users' satisfaction and requirements.
\end{abstract}

Keywords: Key performance indicators (KPIs), network, public services, smart city, right of way, video-to-video (v2v) communication.

\section{Introduction}

Digital Agenda Europe looks to the future for Europe and suggests that due to global competition and demographic ageing, citizens of Europe will have to at least work harder, longer -and with the help of ICT- work smarter to enhance standards of living. The agenda has proposed several policy measures together with applied initiatives to take full advantage of technical, financial and social potential, mainly via the integration of ICTs for exercising business activities, communicating, working and proposing options for entertainment upon the use of Internet as an essential part. In this innovative scope directly affecting market and people's behavior, several pilot 
proposals for open innovation of Internet-enabled services in smart cities have been realized, while several others are actually in progress. Conformant to the above, in the LiveCity ("Live Video-to-Video Supporting Interactive City Infrastructure") PSP-ICT Project (Grant Agreement No.297291) we chose one noteworthy and imminent Internet service, that is the video-to-video (v2v) communication service, and consider several specific applications with various user communities in different European cities to confirm the components constituting the service in parallel with evaluation of the related stakeholder experience and for the preparation of a wider (mass) market deployment.

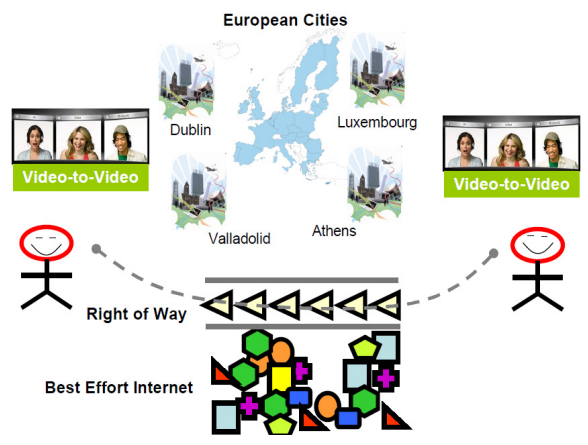

Fig. 1. HD v2v communication in four European cities supported by a globally reachable Right of Way

The public Internet of today can occasionally offer a globally reachable network. We foresee that the Internet of the Future will contain a Right of Way (RoW) for a user's traffic lacking of interference from non-desired traffic (see Fig.1). This kind of RoWs can now be realized in specific virtual private networks (VPNs) using appropriate mechanisms based on the quality of service (QoS); however a right of way with no interference from unwanted traffic is not actually offered to the mass market. For the provision of adequate utility to the users, such RoW has to be reasonably offered at reasonable tariff, must be easily available and has to be supported on the global Internet, if possible. However, inelastic traffic sources such as high-definition (HD) video-to-video are not adequately well supported on the current Internet and live user's video experience can become complicated. The LiveCity project intends to overcome existing difficulties and support the development of appropriate scenarios and/or use-cases, emphasizing upon selective thematic domains where live video communications of high definition and quality could be realized. This is done through the proper structuring and deployment of a wireline and $4 \mathrm{G}$ wireless network of cities, also including a RoW without interference from unwanted traffic, so that each user in any of the involved cities can experience live interactive HD v2v. Thus, LiveCity becomes a pure technology integration trial aiming to provide advanced related $\mathrm{v} 2 \mathrm{v}$ services to more than 3,000 users in four European cities. This unifying approach in the originally involved cities underpins an exploitable platform which can later be rolled out to other cities and communities. 
LiveCity seeks "to bridge the gap" between existing systems and the requirements of various user communities by moving from a set of closed platforms to an "open set" of heterogeneous tools and specifications. Thus, it will transform how people interact online by moving beyond the 2D webpage model to a dedicated platform where people can interact in live streams with one another. The LiveCity approach allows for the development of specific impacts within chosen public sector service domains (i.e.: eHealth, Education, Emergency and City Experiences) and also for the generation of Key Performance Indicators (KPIs) which are relevant within the selected domains. The project consists of a number of key target areas, that is: education, municipal services, e-health, and museum content delivery. Each of these groups consists of a variety of stakeholders (e.g., schools, local government, hospitals, museums) and user communities (e.g.: teachers, doctors, members of the public). User \& stakeholder requirements will be gathered prior to running the pilots to ensure the resulting applications are appropriate, easy to use and future-proofed. In each case a detailed requirements capture phase will be conducted with the relevant parties.

\section{LiveCity Concept and Essential Approach}

The vision of LiveCity is to realize $\mathrm{v} 2 \mathrm{v}$ pilots for different communities of users in the cities of Athens, Dublin, Luxembourg and Valladolid, for a wide diversity of applications and with the aim of sustaining the corresponding network infrastructure with a RoW without interference from unwanted traffic on city networks. The usage of a proper platform for structuring the right of way in the involved cities will lower cost and ameliorate interoperability features, as this approach could also stand as a kind of a common interoperable standard. If reachability is high then value will be high according to Metcalf's law'. By implementing pilots with diverse user communities and by sharing common service platforms and related services and applications in a set of key areas across the partner cities, a common result can be synthesized and disseminated to other European cities. LiveCity is user community driven - and is built for improving core public services such as Education (teachers and school children), Public Administration (municipal administrations in the cities), Education (teachers and school children), Health (ambulances, patients, doctors and nurses) and City Experience (museums, tourist and culture consumers) in the involved target cities. Infrastructure is built by using a dedicated modern network infrastructure, supported by the network operators-partners of the project. The backbone connectivity will be by using a mixture of interconnection methods and the city of Frankfurt will be considered as a "hub". The proposed infrastructure is to be considered as a "real market"-oriented one, with the aim of providing appropriate facilities for the completion of the purposes of the LiveCity project during its lifetime, while identifying issues for a direct deployment and offering of the intended facilities in the market sector, especially to the end users and/or the citizens of the cities involved in the project. To this, we integrate the existing Virtual Path Slice

\footnotetext{
${ }^{1}$ Metcalfe's law states that the value of a telecommunications network is proportional to the square of the number of connected users of the system.
} 
(VPS) controller and a number of ingredients building the infrastructure to support a LiveCity "Living-Lab" scope. Furthermore, LiveCity brings together the value chain and a proper ecosystem able to support $\mathrm{v} 2 \mathrm{v}$, by incorporating end-users communities in the cities, public service providers, network infrastructure providers, technology providers and academic research. Video-to-video is used in some applications today but is often limited by the low image definition, image stuttering and a poor user experience. Much of this limitation is caused by deficient bandwidth available to the application. Widespread deployment of higher speed technologies (e.g. HSPA, LTE, WiMAX) and methods of allocating bandwidth guarantees end-to-end supporting higher quality and live video mean a new range of use cases are becoming available to city citizens.

Since LiveCity seeks to provide real-life services that can be used by a variety of people for several purposes, all these cases require an implicit understanding of the effect that the technology will have on the end-users so as to ensure that they are successfully adopted. Furthermore, the nature of some applications also requires significant stakeholder involvement in the design and evaluation as, without this, the responsible organizations are unlikely to rollout such technologies. To support the above issues, the project will embed users, experts and stakeholders within the design process from an early stage. From an end-user's perspective a process will be adopted, known as "rapid contextual design" that places the system designers in direct contact with end-users so that to capture their usability and functional requirements. Stakeholders will also play a key-role in the design process by being involved while for public exhibits in museums, professional artist(s) will design the installation.

\begin{tabular}{|c|}
\hline Problem Analysis \\
\hline $\begin{array}{l}\text { For each system } 8-12 \text { people will be interviewed to identify their needs for the new } \\
\text { system; this will be partially based on how they undertake their existing work. }\end{array}$ \\
\hline Contextual Interviews \\
\hline $\begin{array}{l}\text { A group of } 8-12 \text { people will be observed and then interviewed at their place of work } \\
\text { undertaking normal daily tasks; in particular those that are potentially relevant for the } \\
\text { v } 2 \mathrm{v} \text { scenarios. }\end{array}$ \\
\hline Interpret and Model Data \\
\hline $\begin{array}{l}\text { Two evaluators will agree on common findings from each data source and a team } \\
\text { meeting will be held to agree on results. Model results will be using appropriate } \\
\text { contextual design approaches. } \\
\text { Presentation of results to selected sample of drivers will take place. }\end{array}$ \\
\hline Consolidation \\
\hline $\begin{array}{l}\text { Consolidation of the results and modeling of data based on existing contextual design } \\
\text { approaches. }\end{array}$ \\
\hline Visioning \\
\hline $\begin{array}{l}\text { Develop a high level view of the problem domain and walk through the results from } \\
\text { the consolidation, in order to "refine" the concepts and develop initial ideals. }\end{array}$ \\
\hline Storyboarding \\
\hline $\begin{array}{l}\text { Groups of } 8-12 \text { end-users will be invited to take part in focus groups that are to be } \\
\text { designed to refine the early level and conceptual phases leading to the development of } \\
\text { user-oriented conceptual designs of the end-systems. These will take the form of } \\
\text { interface mock-ups (suggestions) coupled with storyboarding. }\end{array}$ \\
\hline UI Design and Prototyping \\
\hline $\begin{array}{l}\text { The basic user interface (UI) design will be implemented in prototype form and tested } \\
\text { initially with other researchers then with } 12 \text { potential end users. This will take the } \\
\text { form of interviews and focus groups based around the prototypes. } \\
\text { This stage will be used to identify weaknesses in the overall conceptual design and } \\
\text { early user interface mock-ups. The process will be repeated until the outcomes reach a } \\
\text { satisfactory level, in particular to remove any key functional or usability issues. }\end{array}$ \\
\hline
\end{tabular}

Fig. 2. Contextual design process including an indicative outline of the requirements capture phase, while activating Stakeholders' Committees 
Stakeholder Committees will thus be established to "guide" the pilot actions, per identified scenario (or use-case), and to provide a perspective grounded in the local user community. Depending on each separate case, each committee will include representatives from the involved municipality authority, representatives from the involved school and/or museum (or other cultural institute) and/or hospital, as well as some end-users and (academic or market) experts. Fig. 2 provides a detailed structure of the proposed contextual design process to be adopted for this purpose. A key element of LiveCity is that the systems should be used within specific contexts. Either by individuals or groups of individuals, a critical part of ensuring successful delivery and uptake is an understanding of their motivations and societal aspects, as users' interests, emotions, hopes, passions, fears, and frustrations are important and powerful factors in choosing, learning, and using a technology [1]. As obviously, the project will base its work on ethnographic methods, in particular the use of contextual design ([2], [3]) and activity theory (as in [1] and [4]) coupled a participatory design approaches that involve real users. Indeed, it is important not only to consider the physical design of a device or its interface but also how it relates or integrates into the wider social environment. An outline of the requirements capture phase is contained within Fig.2. Numbers of participants and the structure of each task is drawn from the rapid contextual design approach.

\section{LiveCity Concept Implementation Related to Defined KPIs}

To evaluate the effectiveness of the proposed v2v-based use cases, stakeholder evaluation and key performance indicators will be identified and measured for each specific one. The LiveCity hypothesis is that these indicators can be enhanced with $\mathrm{v} 2 \mathrm{v}$. Other indicators will be collected and evaluated for the different pilots. The innovation evaluation strategy used for assessing the LiveCity platform is distinctive and holistic as it will evaluate technical and user-centric criteria against defined KPIs combining qualitative and quantitative research methods. An adopted balance score card-based analysis will be used to classify and map the defined KPIs against target measurements for both technical and user aspects. This will be combined with a value chain perspective to identify the impact of specific KPIs in relation to other KPIs at different levels of the LiveCity Platform. For example, the value chain perspective will help to identify how and what impact a specific KPI at the network level may have on other KPIs at application or user levels. Such an approach will provide a robust evaluation strategy for the complete LiveCity facility which will verify project results in a measurable way.

As LiveCity entails different pilots in different cities, it is essential that the crossover of best practices and learning are shared between the pilots and synergy is well established. We propose to maintain a "How-to" guide with the purpose of provoking action so that the positive learning becomes embedded in the project's way of working, and that the project avoids any negative lessons. The project will adhere to a communication plan containing a description of the means and frequency of communication between the project partners (internal parties) and other stakeholders 
(external parties). A part on LiveCity's web-portal will be dedicated to maintaining the daily quality log. The frequency of quality reviews will be established for each pilot, outputs of which together with the daily quality log will form the lessons learned (i.e.: "How-to" guide) for LiveCity. This guide will be a living document owned by all project partners. Any updates from the quality review or daily quality $\log$ will be notified to all project partners and reflected in the website.

\subsection{Process for the Definition of Technical and Usability KPIs}

LiveCity intends to apply a set of technical and non-technical (usability) KPIs that will facilitate the evaluation of the related platform. To this end, two expert steering committees will be appointed to oversee the development, evaluation and refinement of KPIs both at technical and usability levels. In this respect, work is to be performed in two main phases. The first phase will consist of two stages: (a) Developing of a set of initial, verifiable and quantified KPIs which will be used for evaluating LiveCity architecture, applications and user experience, and; (b) making sure that stakeholders' (users, network and service providers) requirements (e.g.: usability, social, economic, legal, political and technological) are properly reflected in the design-implementation of platform. The second phase will involve applying these revised KPIs and usability metrics in the final pilot of the platform by assessing and revising KPIs (technical and usability metrics) on the basis of the evaluation of the LiveCity platform. Results of these will form the basis of the final recommendations and produce a "How-to Guide" for designing and implementing v2v infrastructure \& applications. The related work is to be realized in a process comprising of six distinct "stages", as identified below:

The first "stage" of the proposed approach will involve conducting a comprehensive search of normative and secondary research to formulate an initial set of KPIs for evaluating v2v technology. Thus, KPIs will be categorized according to technical (architecture and application) and non-technical (user-centric) criteria and will provide the agenda for further empirical work. The second "stage" of the intended effort will focus on the requirements analysis for LiveCity evaluation and will involve collecting both technical and usability requirements from different stakeholders. Focus groups with the network, application \& service providers and with potential user communities for the scheduled pilot actions will be used to identify requirements for each specific pilot and compile them into measurable and verifiable requirements that will be then translated into KPIs. A balance score cardbased analysis will be adopted for the data collected in the network and application layers of the platform together with user experiences to enable the definition of the technical and non technical KPIs in the subsequent stage of analysis. The third "stage" will involve developing initial KPI metrics based on the output from the previous stages. It is envisaged that the KPIs will contain the following dimensions (as explained in sections 3.2-3.4) to evaluate network, application and process level performance of the LiveCity platform.

The fourth "stage" will focus on the development of KPI metrics for user experience, when using the LiveCity applications. Some initial KPIs examples are: 
- Compatibility: The system should be based on a familiar hand-held device (camera/video image transmitter) or standard screen, but should also be designed with other space-constrained devices, such as mobile phones in mind.

- Document Design: The layout and interactivity of the system should be dynamic, simple to use and helpful to the user.

- Usability and Ease of Use: The system should be easy to understand, efficient to use, easy to interact and remember, subjectively pleasing and have clear help instructions.

- Efficiency: The accuracy and completeness, with which users can achieve specific goals in the easiest way, produce better output, reduced time taken to perform a task and increase job performance.

- Preventing loss of Information: The system should prevent (and/or minimize) any instances where information may get lost.

- Response Time: System's response time or average waiting time between a user action; system's response time should be acceptable (time will be predefined).

- Interaction Time: The average interaction time required to complete a certain action before $\mathrm{v} 2 \mathrm{v}$ content is delivered to the hand-held device/screen/mobile set.

- Number of Interactions Per Service Request: Average number of interactions required to complete a certain action.

- Level of Awareness: The strategy used to raise the awareness of the system in terms of adoption. This should include training and familiarizing with the system.

- Improvement of User Experience: Perceived efficiency in improvement of the user experience when using the system.

- The Interface in terms of Appearance: The layout of the system should be simple to view, pleasing to the eye and easy to navigate.

- Usefulness: The system should be useful to the user when interacting.

- Comments and Command lines: The system should have features to allow navigation and easy access of content.

The subsequent fifth "stage" will evaluate LiveCity technology against the developed KPIs with each user pilot (i.e.: health, education and municipality). The activities will focus on collecting evidences for each of the KPIs during the pre-pilot and pilot stage of the LiveCity platform. For platform-level KPIs, the team will use logging files from the network and application platforms. For collecting the evidences of the user pilots, the team will use structured questionnaires/case report forms (CRFs) which will be distributed to all participants in user pilots. Collected KPI data will be monitored \& entered into dedicated datasets by database templates (e.g., Excel) and analyzed by descriptive and exploratory statistical methods using routine statistical software packages (e.g.: SPSS, STATA, etc.). This will work closely with the rest of the Work Packages to ensure that the required standards are achieved in the user pilots by performing the required evaluation cycles (e.g., illustrations/diagrams for comparative purposes will be generated and analysed).

The final sixth "stage" will involve compiling the data generated from "stage 5" in both the pre-pilot and pilot stages as well as producing a "How-to guide" for v2v. Work here will identify whether KPIs for the platform (network, application and user 
pilots) are satisfied and where recommendations for further improvements to the developed prototypes/services need to be made as advised by the steering committee. In this respect, refinements to the initial KPIs after the pre-pilot and further improvements will be recommended where appropriate, for future reference. As LiveCity entails different pilots in different cities, it is essential that the cross-over of best practices and learning are shared between pilots and synergy is established. We propose to maintain a "How-to guide" with the purpose of provoking action so that the positive learning becomes embedded in the project's way of working, and that the project avoids any negative lessons.

\subsection{KPIs for Network Right of Way and Application Right of Way}

In this context, KPIs will be identified for assessing the quality of experience (QoE). An adapted service level agreement (SLAs) with the relevant service providers will be maintained by the carrier for the RoW invoked by the requirements of the various applications. Other network RoW-based KPIs that will be measured will focus per specific thematic category, as shown in Table 1.

Table 1. Network Right of Way- based KPIs

\begin{tabular}{|c|c|c|}
\hline Category & Parameter & Description/Example \\
\hline \multirow{4}{*}{ Scalability } & 1. Set up time & $\begin{array}{l}\text { Set up time for a VPS to implement the ROW. } \\
\text { The set up time has to be of the order available in } \\
\text { the PSTN or GSM networks ( } 1 \text { Second })\end{array}$ \\
\hline & $\begin{array}{l}\text { 2. Concurrent Session } \\
\text { per processing node }\end{array}$ & $\begin{array}{l}\text { Ability of the Future Internet Right of Way } \\
\text { Control Plane to scale to high volumes. }\end{array}$ \\
\hline & $\begin{array}{l}\text { 3. Busy Hour Call } \\
\text { Attempt }\end{array}$ & $\begin{array}{l}\text { Achieve dynamic virtual path establishment at } \\
\text { large scale (e.g. an autonomous system could have } \\
\text { upwards of } 10 \mathrm{~m} \text { users on a network). }\end{array}$ \\
\hline & 4. Bandwidth & Bandwidth requested vs measured at high scale. \\
\hline \multirow{5}{*}{ Reliability } & $\begin{array}{l}\text { 1. Mean time to } \\
\text { failure (MTTF) }\end{array}$ & Normal operation time between failures. \\
\hline & $\begin{array}{l}\text { 2. Mean time to repair } \\
\text { (MTTR) }\end{array}$ & $\begin{array}{l}\text { Down time from failure to restarting normal } \\
\text { operation. }\end{array}$ \\
\hline & $\begin{array}{l}\text { 3. Mean time between } \\
\text { failures (MTBF) }\end{array}$ & MTBF=MTTF+MTTR \\
\hline & $\begin{array}{l}\text { 4. Percentage of time } \\
\text { available }\end{array}$ & MTTF/(MTTF+MTTR) \\
\hline & $\begin{array}{l}\text { 5. Loss or corruption } \\
\text { rate }\end{array}$ & $\begin{array}{l}\text { Proportion of total data that does not arrive as } \\
\text { sent, e.g., network error rate, packet loss. }\end{array}$ \\
\hline \multirow{3}{*}{ Security } & 1. Authorisation & $\begin{array}{l}\text { E.g. types of credentials are passwords and digital } \\
\text { certificates. }\end{array}$ \\
\hline & 2. Authentication & E.g. IP filtering and tunneling \\
\hline & 3. Accounting & E.g. nature of service delivered and status report. \\
\hline
\end{tabular}


It is critical to the success of the pilots to ensure that the network connections and software inter-work correctly. Correct functioning of the service must be individually checked, before the user pilots begin. Related KPIs include:

Table 2. KPIs for Application Right of Way

\begin{tabular}{|l|l|}
\hline Category & Description/Example \\
\hline $\begin{array}{l}\text { Concurrent } \\
\text { Connection }\end{array}$ & $\begin{array}{l}\text { Ensure that the application is compatible with various } \\
\text { communication channels and protocols (Wi-Fi, sDSL, HSPA, LTE, } \\
\text { FTTO). }\end{array}$ \\
\hline $\begin{array}{l}\text { Variability of the } \\
\text { Device }\end{array}$ & $\begin{array}{l}\text { Ensure that the application will run on various devices that will be } \\
\text { used for the LiveCity platform. To facilitate this, KPIs for this } \\
\text { category will be based on an adaptive content management system. }\end{array}$ \\
\hline $\begin{array}{l}\text { Support for } \\
\text { Codecs }\end{array}$ & $\begin{array}{l}\text { Ensure that the application supports the various codecs across } \\
\text { different devices. }\end{array}$ \\
\hline Scalability & $\begin{array}{l}\text { Ability of v2v Social Network Platform to provide a high number } \\
\text { of concurrent v2v sessions. }\end{array}$ \\
\hline
\end{tabular}

\subsection{KPIs for User Pilots}

The following KPIs for the user pilots will be discussed-verified with the leading partners of each case (health, municipality and city experience). Some initial and indicative examples of KPIs are as follows:

Health Experience use cases may relate to the following:

(a) KPIs for Emergency department - ambulance services (ED) use case: since three different patient populations by disease type will be approached, this use-case will explore the following KPIs:

- for heart attack: time on scene; time to diagnostic electrocardiogram (ECG); time to thrombolysis (clot busting); time to cardiac catheterisation laboratory; length of hospital stay;

- for stroke: time on scene; time to CT (Computed Tomography); time to stroke physician; time to thrombolysis; neurological functioning; survival; length of hospital stay; return to self care;

- for trauma entrapment: time on scene; time to analgesia; time to extrication; time to trauma team; time to theatre (if relevant); trauma score; survival.

(b) KPIs for Medical education use case: Multiple-choice questions score (pre- and post-module MCQ and problem-based cases to assess medical students learning); evaluation scores (forms filled in by healthcare professionals and medical students following the module); cost effectiveness (CE) ratio / cost-effectiveness analysis (CEA) for evaluation in terms of comparing the existing, mainly small group teaching practice versus the new, video-link (v2v) interactive teaching.

(c) KPIs for Preventive health / smoking cessation use case: Pre- and post-case smoking rates (\%), chemically validated (continine) smoking cessation rates at term, relapse rates (\%) versus pre-case (if available), patient satisfaction/ views on intervention (qualitative).

(d) KPIs for patients' treatment tele-monitoring (TM) use case: quality of life measures; wellbeing score; treatment satisfaction. 
Education use cases may implicate skills tests; knowledge tests; attitudinal aspects; learning experience, overall performance.

Municipality and City Experience use cases. This considers wellbeing; service satisfaction; usefulness; speed of resolving queries.

\subsection{Examples of KPIs for the Field Trials}

A set of generic KPI will also be used for the user pilots to ensure that the field trails are completed successfully. These can take into account of the following: (i) Situational and positional awareness: Here the facilitators of the user pilots need to take appropriate steps to increase the awareness of the LiveCity system by educating the users on the technologies-equipment and applications-interface that will be used during the field trial, together with increasing user awareness about the benefits \& advantages of the system; (ii) Availability and connectivity: Connection between the LiveCity user interface and the network should be fast and error free; (iii) Data security \& privacy to ensure secure transmission of $\mathrm{v} 2 \mathrm{v}$ content over various wireless technologies; (iv) Data protection to ensure authentication and authorization for access to v2v content; (v) Reliability: The system should always and consistently show the correct images in high quality video and transmit the right information for a given use case scenario; (vi) Prevent the misinterpretation of information: The system should prevent and minimise any instances where information may get misinterpreted.

\section{$4 \quad$ Summary and Conclusion}

This paper has highlighted the aim and objectives of the LiveCity project funded under the European Commission's $7^{\text {th }}$ framework ICT-PSP context. The paper has discussed how the LiveCity project intends to develop and implement a v2v platform across some European cities for a range of diverse applications. By using a common network infrastructure platform for building the right of way in the target cities, costs can be reduced and services can be improved in core areas such as health, education, city experience and municipality services. We have also proposed a scope for the development of appropriate KPIs to evaluate the related platform development from various points of view and aspects per use case.

\section{References}

1. Kaptelinen, V., Nardi, B.: Acting with Technology: Activity Theory and Interaction Design. Amazon Media sarl (Kindle Edition) (2006)

2. Beyer, H., Holtzblatt, K.: Contextual design: designing customer-centered systems. Morgan Kaufmann Publishers (1998)

3. Holtzblatt, K.: Rapid Contextual Design: A How-to Guide to Key Techniques for UserCentered Design (Interactive Technologies). Morgan Kaufmann (2004)

4. Nardi, B.: Context and Consciousness: Activity Theory and Human-Computer Interaction. MIT Press (1995) 